\title{
Hiperactividad simpática paroxística en lesión neurologica: revisión
}

Mónica Patricia Herrera Martínez ${ }^{1,2}$, Romario Mendoza ${ }^{1,2}$, Winston Cardenas ${ }^{1,2}$, María Paula Maza Moscote-Salazar ${ }^{1,2}$, YanCarlos Ramos ${ }^{4}$, Luis Rafael Moscote-Salazar ${ }^{2,3}$

1 Estudiante de Medicina. Universidad de Cartagena, Cartagena de Indias, Colombia.

2 Cartagena Neurotrauma Research Group, Centro de Investigaciones Biomédicas (CIB), Facultad de Medicina, Universidad de Cartagena, Cartagena Colombia.

3 Médico. Especialista en Neurocirugía. Faculta de Medicina, Universidad de Cartagena. Cartagena de Indias, Bolívar.

4 Médico, Universidad de Cartagena, Cartagena de Indias, Colombia.

Rev. Chil. Neurocirugía 45: 67-72, 2019

\section{Resumen}

La hiperactividad simpática paroxística es un fenómeno que se presenta en individuos, independientemente de su edad y sexo, posterior al daño cerebral. Es una respuesta fisiológica donde se ve actividad aumentada del sistema nervioso autónomo simpático, pero a su vez existe influencia hormonal, que se evidencia en las manifestaciones clínicas del paciente. Los neurocirujanos debemos identificar esta alteración en nuestros pacientes de una manera oportuna.

Palabras clave: Hiperactividad simpática paroxística, daño cerebral, hormonas.

\begin{abstract}
Paroxysmal sympathetic hyperactivity is a phenomenon that occurs in individuals, regardless of age and sex, after brain damage. It is a physiological response where increased activity of the sympathetic autonomic nervous system is seen, but in turn there is hormonal influence, which is evidenced in the clinical manifestations of the patient. Neurosurgeons must know and identify this alteration in our patients in a timely manner.
\end{abstract}

Key words: Paroxysmal sympathetic hyperactivity, brain damage, hormones.

\section{Introducción}

La hiperactividad simpática paroxística (HSP), descrita desde 1929 por Wilder Penfield $^{1,2}$ es una urgencia neurológica potencialmente letal secundaria a lesiones cerebrales ${ }^{3}$, tales como trauma craneoencefálico, encefalopatía anóxica-isquémica, ataque cerebrovascular ${ }^{2,3}$, encefalitis $^{4}$, ahorcamiento ${ }^{1}$, entre otras. La HSP se ha descrito como un síndrome caracterizado por diaforesis, taquicardia, taquipnea, hipertensión, posturas motoras anómalas e hipertermia ${ }^{1,3,5-7}$ en respuesta a la sobre-activación del sistema nervioso simpático y descarga hormonal ${ }^{6}$. Su incidencia está estimada en un intervalo desde $7,7 \%$ a $33 \%$ de los pacientes que se admiten en la unidad de cuidados intensivos generalmente posterior a un trauma craneoencefálico ${ }^{1,7,8}$, y su diagnóstico se basa en la sospecha clínica y exclusión en el diagnóstico diferencial ${ }^{3}$, aunque cabe resaltar que entre las complejidades diagnósticas está el comienzo insidioso de los síntomas, la falta de parámetros medidos o la fisiopatología pobremente clarificada (sobre todo en población infantil donde la bibliografía es muy limitada $)^{4,7}$, y esto además impacta en el pronóstico neurológico del paciente ${ }^{2}$, la estancia hospitalaria y costos en los servicios $^{4,7}$.

\section{Nomenclatura}

La HSP ha sido descrita con más de 30 términos $^{9}$ registrados como disautonomía central, tormenta simpática, inestabilidad autonómica paroxística con distonia, entre otros ${ }^{6}$ pero el término que en consenso se usa para referirse es "Hiperactividad simpática paroxística" 
por ser específico, no conllevar en él otra patología subyacente y ser acorde a la definición de este síndrome ${ }^{9}$.

\section{Fisiopatologia de la HSP}

Hasta el día de hoy, los acontecimientos que dan lugar al desequilibrio de la producción y liberación de las catecolaminas, hecho que caracteriza a la HSP, no están completamente claros ${ }^{10}$. Las primeras teorías que buscaban darle explicación a este fenómeno proponían que las oleadas simpáticas eran desencadenadas por crisis epilépticas o secundarias al síndrome de hipertensión intracraneal ${ }^{3}$. Si bien es cierto que las crisis convulsivas pueden provocar disfunción autonómica, los medicamentos anticonvulsivantes tienen muy poca eficacia como tratamiento de la HSP ${ }^{3,10}$. Estas posturas basadas en mecanismos epileptogénicos carecieron de evidencia en modelos experimentales. Teorías más recientes hacen referencia a que existen interacciones inhibitorias entre centros superiores del sistema nervioso central (como la ínsula y la corteza cingulada) y los centros inferiores hipotalámicos, diencefálicos y del tallo cerebral, los cuales son responsables del control supraespinal del tono simpático ${ }^{11}$. Cuando se presenta una lesión cerebral estas conexiones pueden perderse conllevando a un flujo simpático desmedido $0^{3,10,11}$. Este modelo, aunque explica parte de la HSP, no brinda una explicación completa a todas las características que en ella se presentan. Por otro lado, existe evidencia histopatología y radiológica que sugiere que en pacientes con trauma cerebral severo que han presentado HSP a menudo existe daño en las zonas bajas del tallo cerebral, por lo que es muy poco probable que la actividad de las vías simpáticas que provengan de esos lugares explique completamente este fenómeno ${ }^{10}$.

El modelo más reciente de relación excitación-inhibición reconoce la naturaleza inhibitoria de los centros localizados en el diencéfalo y el tallo cerebral, donde cumplen una función neuromoduladora limitando la sensibilización y la amplificación de las aferencias provenientes de la medula espinal $\left.\right|^{3,12}$. Esta teoría sugiere un proceso de dos etapas, donde la desconexión de las vías inhibitorias conlleva a la excitación de los circuitos medulares ${ }^{13}$. La plasticidad de estas vías produce la amplificación de los estímulos leves, que en última instancia produce alodinia ${ }^{14}$. Este concepto se refiere a la percepción inadecuada de dolor con estímulos sensitivos leves. De esta manera, después de una lesión cerebral, un estímulo nocivo o no nocivo puede tener la capacidad de inducir un "feedback" positivo con el potencial para producir el fenómeno de HSP. Estos estímulos incluyen intervenciones comunes como el cambio de posición, los baños, entre otros ${ }^{10,11}$

\section{Criterios diagnósticos}

Uno de los requerimientos para realizar el diagnóstico de HSP es tener una sospecha clínica elevada, especialmente, en los pacientes que hayan sufrido un trauma cerebral ${ }^{2,3,15}$. Debido que el diagnóstico de HSP se basa en los hallazgos clínicos, los exámenes complementarios no son los instrumentos necesarios para detectar con certeza esta condición ${ }^{3}$. Además, en los diferentes estudios realizados, los criterios para diagnosticar la HSP han sido muy heterogéneos, dificultando este proceso ${ }^{16}$. Sin embargo, en el año 2014 se estableció una herramienta cuyo objetivo fue estandarizar estos criterios a modo que pueda emplearse en los centros asistenciales como método diagnóstico y orientación al manejo clínico de la HSP; esta herramienta se conoce como Medida de Evaluación de PSH (PSH-AM, por sus siglas en inglés). LA PSH-AM posee dos componentes: 1) la herramienta de Escala de Características Clínicas (CFS) y 2) la Herramienta de Probabilidad Diagnóstica (DLT) ${ }^{1,9,11,17}$. La CFS incorpora las manifestaciones clínicas que se presentan con mayor frecuencia en la HSP (frecuencia cardíaca, frecuencia respiratoria, temperatura, presión arterial sistólica, gravedad de la diaforesis y de la postura motora), puntuando cada categoría de 0 a 3 , donde el valor total puede oscilar entre 0 (sin síntomas) hasta 18 (síntomas más graves de HSP) $1,3,9,11,17$.

La DLT, por su parte, es utilizada para la exclusión de posibles diagnósticos diferenciales y su valor total varía entre 0 y 11 , donde esta última puntuación representa la máxima probabilidad para HSP. En esta herramienta se encuentran 11 criterios donde se busca evaluar: el tiempo que se mantienen las manifestaciones, la cantidad de episodios por día, el número de días consecutivos que el paciente tiene síntomas similares a PSH, los fármacos administrados por características simpáticas, los posibles diagnósticos diferenciales y otras etiologías probables de las características ${ }^{1,3,9,17}$.

Por último, se suman diariamente los puntajes totales de cada herramienta y se calcula un promedio por el número de días en que sea evaluado el paciente. De acuerdo a los resultados totales se puede determinar la probabilidad de padecer HSP, clasificándola en: 1) "improbable" (puntuación < 8), 2) "posible" (puntuación 8-16) o "probable" (puntuación $>17)^{1,3}$.

\section{Diagnósticos diferenciales}

Es de vital importancia determinar con certeza el diagnóstico de HSP, este proceso debe basarse en una exclusión de diagnósticos diferenciales ${ }^{2,10,17,18}$ debido que las manifestaciones clínicas generadas por esta condición guarda estrecha relación con otras condiciones como: convulsiones, bacteriemia, sepsis, tromboembolismo pulmonar, abstinencia de alcohol o drogas, síndrome serotoninérgico, síndrome neuroléptico maligno, suspensión de opioides o sedantes, hipertensión intracraneal, hidrocefalia, agitación, dolor, entre otras ${ }^{3,10,15,17-20}$. Además, algunos de estos diagnósticos pueden acompañar la HSP, e incluso complicar su manejo ${ }^{19}$.

\section{Influencia de hormonas}

En diversos estudios, los valores elevados de catecolaminas o de otras hormonas, como las adrenocorticales, se han asociado a lesión traumática cerebral $^{2,16}$. Dicho aumento de catecolaminas es proporcional a la gravedad del daño neurológico, lo cual puede relacionarse con la sintomatología de la $\mathrm{HSP}^{2}$. Sin embargo, la concentración de catecolaminas no es un criterio absoluto de HSP 17,20 .

Existen diversas investigaciones en las cuales se puede evidenciar la relación entre HSP y catecolaminas periféricas, y posiblemente la liberación de corticosteroides $^{3,11}$. En una de esas investigaciones se han comparado los valores de catecolaminas en un paciente con HSP en estado de reposo y a la hora de los paroxismos y se encontraron elevaciones de norepinefrina en un 60 y epinefrina en un $100 \%$ con respecto 
a los valores basales. Sin embargo, no se ha evaluado el impacto de los episodios de HSP en los ejes de hormonas tiroideas o adrenocorticales ${ }^{16}$.

\section{Tratamiento}

El manejo de la hiperactividad simpática paroxística es considerado un desafío en la práctica clínica, representa un reto para los profesionales de la salud, que deben hallar un equilibrio entre el tratamiento oportuno de los síntomas, la elección de los fármacos y sus dosis adecuadas, e impedir los efectos secundarios $^{2,3,19}$. El tratamiento de la HSP requiere una combinación de un tratamiento farmacológico y no farma- cológico $\left.{ }^{1}\right)$. Enfocado en el control de los síntomas, para llevar a cabo esto, se emplean tres medidas: primero, inhibir el flujo simpático central, Segundo, inhibir proceso sensorial aferente para impedir el desarrollo de alodinia. Y tercero, bloquear las respuestas de los órganos efectores del sistema nervioso simpático $3,10,11,20$.

En el tratamiento no farmacológico se debe identificar si los síntomas son producidos por estímulos secundarios, como lo son tocar, girar o aspirar con tubo endotraqueal, y se debe hacer un esfuerzo para minimizar la aparición de los síntomas ${ }^{19}$. Es importante mencionar el papel de la nutrición y la fisioterapia en el manejo. En la literatura se han reportado series de casos que eviden- cian un aumento de los requerimientos calóricos en los pacientes con HSP, algunos, muestran pérdidas superiores al $20 \%$ de su peso corporal, por esto la intervención nutricional es necesaria para una disminución de la morbilidad. La fisioterapia en la atención de la movilidad, para la rigidez y prevenir las contracturas musculares ${ }^{11,21}$.

\section{Tratamiento farmacológico}

El manejo médico abarca diversos tipos de fármacos, como lo son, los $\alpha 2$ agonistas, $\beta$-bloqueantes, benzodiazepinas, agonistas dopaminérgicos, opioides, agentes GABAérgicos, y dantrolene ( $\mathrm{Ta}$ bla 1$)^{2,3,19,20}$. En este momento, en el tra-

Tabla 1.

Medicamentos utilizados en el tratamiento de la HSP $2,3,11,20$

\begin{tabular}{|c|c|c|}
\hline Medicamento & Sitio de acción & Reducción de síntomas \\
\hline \multicolumn{3}{|l|}{$\alpha 2$ Agonistas } \\
\hline Clonidina & $\begin{array}{l}\text { Adrenoceptores } \alpha 2 \text { en el cerebro y la médula } \\
\text { espinal }\end{array}$ & Hipertensión, taquicardia \\
\hline Dexmedetomidina & $\begin{array}{l}\text { Adrenoceptores } \alpha 2 \text { en el cerebro y la médula } \\
\text { espinal }\end{array}$ & Hipertensión, agitación, taquicardia \\
\hline \multicolumn{3}{|l|}{$\beta$-bloqueadores } \\
\hline Propanolol & $\begin{array}{l}\text { Adrenoceptores } \beta \text { no selectivos (centrales, } \\
\text { cardíacos y periféricos) }\end{array}$ & Hipertensión, taquicardia, fiebre \\
\hline \multicolumn{3}{|l|}{ Opioides } \\
\hline Morfina & $\begin{array}{l}\text { Receptores } \mu \text { - opioides en el cerebro, mé- } \\
\text { dula espinal y periferia }\end{array}$ & $\begin{array}{l}\text { Respuesta a la alodinia, vasodilatación periferica, } \\
\text { taquicardia }\end{array}$ \\
\hline Fentanilo & $\begin{array}{l}\text { Receptores } \mu \text { - opioides en el cerebro, mé- } \\
\text { dula espinal y periferia }\end{array}$ & $\begin{array}{l}\text { Respuesta a la alodinia, vasodilatación periferica, } \\
\text { taquicardia }\end{array}$ \\
\hline \multicolumn{3}{|l|}{ Benzodiacepinas } \\
\hline Midazolam & $\begin{array}{l}\text { Receptores centrales en complejos GABA } \\
\text { en cerebro y médula espinal }\end{array}$ & Agitación, ansiedad, hipertensión, y taquicardia \\
\hline Diazepam & $\begin{array}{l}\text { Receptores centrales en complejos GABA } \\
\text { en cerebro y médula espinal }\end{array}$ & Agitación, ansiedad, hipertensión, y taquicardia \\
\hline Lorazepam & $\begin{array}{l}\text { Receptores centrales en complejos GABA } \\
\text { en cerebro y médula espinal }\end{array}$ & Agitación, ansiedad, hipertensión, y taquicardia \\
\hline \multicolumn{3}{|c|}{ Agonistas dopaminergicos } \\
\hline Bromocriptina & Agonista receptor D2-dopamina & Distonia, fiebre \\
\hline \multicolumn{3}{|l|}{ GABAergicos } \\
\hline Gabapentina & Agonista GABA & Respuesta a la alodinia, espasticidad \\
\hline Baclofeno & Agonista específico de GABA B & Dolor, rigidez, clonus \\
\hline \multicolumn{3}{|c|}{ Bloqueantes de la liberación de calcio } \\
\hline Daltrolene & $\begin{array}{l}\text { Receptores de rianodina en el reticulo sar- } \\
\text { coplasmico }\end{array}$ & Postura distónica y espasmos musculares \\
\hline
\end{tabular}


tamiento de los pacientes se requiere la combinación de diversos fármacos para el manejo de los síntomas y prevenir los potenciales efectos secundarios ${ }^{3,19}$.

Los $\alpha 2$ agonistas, actúan en los adrenoceptores $\alpha 2$ ubicados en el cerebro y la médula espinal ${ }^{11}$. El tratamiento de la hiperactividad simpática paroxística abarca el uso de clonidina, ya que reduce el flujo simpático central, es considerada como medicamento de primera línea para el manejo de la hipertensión, sin embargo, es ineficaz para contrarrestar las otras manifestaciones, por esto, su uso requiere combinación con otros fármacos ${ }^{2,10}$. La dosis de clonidina es $0,1-0,3 \mathrm{mg}$ cada $12 \mathrm{~h}$, y sus principales efectos secundarios son incluyen hipotensión, sedación, depresión, y bradicardia $^{3,10,19,20}$. La dexmedetomidina es un agonista $\alpha 2$ intravenoso efectivo en el control de la taquicardia, hipertensión agitación ${ }^{2,19,20}$. Debe ser utilizada en una $\mathrm{UCl}$, y puede usarse en pacientes que no dependen de la ventilación mecáni$\mathrm{ca}^{2,19}$. En 2017, Tan Q. et al., realizaron un estudio retrospectivo que afirma que el uso de dexmedetomidina tiene un efecto preventivo de HSP en pacientes con lesión traumática cerebral severa intervenidos quirúrgicamente, comparado con los sedantes tradicionalmente utilizados $^{22}$

Los $\beta$-bloqueadores disminuyen el efecto de las catecolaminas circulantes. En la HSP son el pilar fundamental del tratamiento de la hipertensión, taquicardia, hiperpirexia y diaforesis. El propanolol es el $\beta$-bloqueador más utilizado por ser lipofilico y atravesar la barrera hematoencefalica, es eficaz en el manejo de HSP, debido a que produce disminución de la presión arterial, frecuencia cardíaca, gasto cardíaco y consumo de oxíge$\mathrm{no}^{2,10,20}$. Schroeppel et al., demostraron que el uso de propranolol en pacientes con lesión cerebral traumatica, disminuye la mortalidad comparado con el uso de otros $\beta$-bloqueadores ${ }^{23}$.

La morfina y el fentanilo son potentes agonistas del receptor $\mu$-opioide en el cerebro, medula espinal y periferia, que aumentan los efectos colinérgicos, e inducen la liberación de histamina produciendo vasodilatación periférica ${ }^{2,11,20}$. Se utilizan para controlar los episodios de crisis, y la morfina para terapia de mantenimiento es usada como un agente oral: oxicodona ${ }^{19}$. Su eficacia es dependiente de la dosis, por tal razón, se debe tener presente los efectos secundarios como la depresión respiratoria, sedación e hipotensión ${ }^{20}$.

Las benzodiazepinas son agonistas del receptor GABA utilizados en el tratamiento de HSP, que contrarrestan la taquicardia, hipertensión, ansiedad y agitación ${ }^{2,11}$. Las benzodiacepinas de acción corta son empleadas en el curso temprano y episodios severos de $\mathrm{HSP}^{2,20}$. El midazolam, lorazepam y diazepam interaccionan con los receptores GABA A, y generan relajación muscular y ansiolisis durante las crisis de HSP. Por esto, se utilizan en el manejo de la distonia y la espasticidad ${ }^{10,19}$.

La bromocriptina es un agonista de dopamina que estimula los receptores D2. Su uso es para el manejo de la distonia y la fiebre, para promover su eficacia, se combina con otros fármacos, como la morfina $^{2}$. Entre sus efectos secundarios se encuentran la discinesia, agitación y confusión ${ }^{20}$. Es importante aclarar, que anteriormente los antagonistas dopaminergicos como la clorpomazina y el haloperidol eran usados en el tratamiento de HSP, pero se evidenció que producen un empeoramiento de los síntomas, por esto, no se recomienda su uso ${ }^{2,20}$.

El baclofeno es un agonista específico del receptor GABA B, cuya acción es reducir la frecuencia e intensidad de los espasmos, alivia el dolor, el clonus y la rigidez muscular ${ }^{2,20}$. El uso de la infusión intratecal de baclofeno (ITB) en dosis de 100-500 $\mu \mathrm{g} /$ día mejoró los síntomas de HSP. A pesar de esto, es un procedimiento costoso, invasivo y no siempre está disponible. Sus efectos secundarios son debilidad muscular, elevación de las enzimas hepáticas, y sedación ${ }^{20}$. Entre las complicaciones se encuentran la infección del LCR, y fallas en el catéter o en la bomba ${ }^{19}$. La gabapentina es un análogo del receptor GABA, útil en el manejo de la espasticidad y el temblor, con una dosis inicial de $300 \mathrm{mg}$ cada 8 horas $^{2,20}$. Es más eficaz que la BIT cuando son utilizados simultáneamente ${ }^{20}$.

El daltrolene es un relajante muscular que impide la liberación de ion calcio del retículo sarcoplásmico en las células del músculo esquelético. Es eficaz en el manejo de la postura distónica. Puede causar hepatotoxicidad y depresión respiratoria, por esto, es necesario el monitoreo de la función hepática previo y durante el tratamiento $0^{2,20}$.

Actualmente, el tratamiento farmacológico está orientado a la suspensión de la crisis, la prevención de los síntomas, $y$ el tratamiento refractario ${ }^{3,19}$. El objetivo en la suspensión de la crisis, es el control del episodio y evitar su progresión, se utilizan fármacos de acción rápida y vida media corta, estos abarcan la morfina, las benzodiacepinas, el propanolol, y su elección depende del síntoma predominante ${ }^{3,17,19}$. En la prevención de los síntomas, el propósito es disminuir los episodios en número, duración e intensidad, para esto, se incluyen fármacos como, el propanolol, $\alpha 2$ agonistas, GABAergicos y benzodiacepinas. El tratamiento refractario, es aquel que se utiliza cuando la HSP no responde al tratamiento y puede tener graves implicaciones como lo son edema pulmonar, daño cardiaco u lesión cerebral secundaria al aumento en la presión arterial. Por esto, se debe administrar fármacos intravenosos, tales como las benzodiacepinas, propofol, opioides y, $\alpha 2$ agonistas (Figura $1)^{3,17,19}$

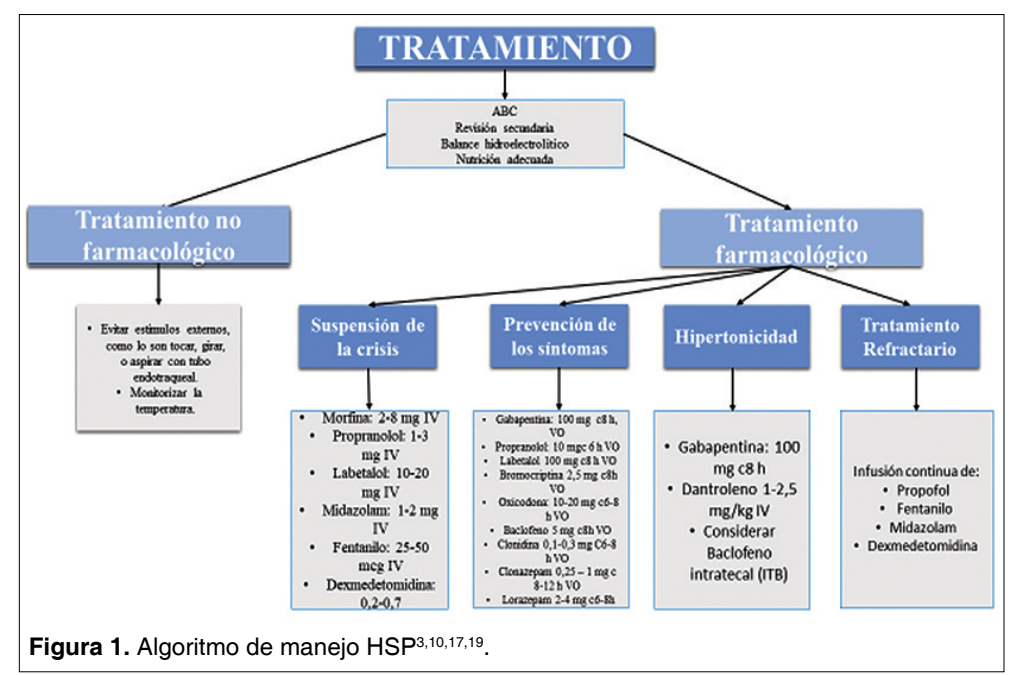




\section{Terapia de oxígeno hiperbárico}

Debido a que todos los medicamentos anteriormente mencionados tienen efectos secundarios, y que para evidenciar mejoría en la sintomatología es necesario la combinación entre ellos. Aparece la terapia de oxigeno hiperbárico (TOHB) como una alternativa de tratamiento, consistente en la inhalación de oxígeno al $100 \%$ en un entorno con la presión atmosférica más alta de lo normal. Debido a su capacidad de aumentar la disponibilidad de oxigeno cerebral, y optimizar el metabolismo aeróbico cerebral después de una lesión, puede mejorar la funcionalidad y disminuir la mortalidad en pacientes con lesión cerebral severa ${ }^{20}$.

En estos pacientes, la TOHB ha sido utilizada luego de un tratamiento farmacológico con resultados insatisfactorios. El tratamiento consiste en ciclos de una vez al día durante 10 días consecutivos, los síntomas se resolvieron luego de 3-10 ciclos. Una serie de casos de China, reportó el probable efecto positivo de la TOHB sobre los paroxismos y las posturas en pacientes con HSP subagudo precoz, después de efectos insatisfactorios con el manejo farmacológico ${ }^{11}$. Aunque la terapia de oxigeno hiperbárico no ha sido aceptada universalmente y existe controversia sobre el pronóstico a largo plazo, puede ser una alternativa que merece ser estudiada, especialmen- te como un tipo de terapia adyuvante que en el futuro permita mayor bienestar para los pacientes ${ }^{20}$.

Por último, es necesario realizar un protocolo del manejo de la hiperactividad simpática paroxística, que estipule la base de un tratamiento oportuno e intensivo, para abordar adecuadamente los pacientes, prevenir complicaciones, disminuir la estancia hospitalaria y mejorar sus posibilidades de rehabilitación ${ }^{3}$. Sin olvidar que, se debe tener claro que para lograr avances significativos para un tratamiento efectivo de la HPS se requiere una mejor comprensión de la base fisiopatológica que sustenta los síntomas ${ }^{16}$.

\section{Cuidado familiar}

La hiperactividad simpática paroxística es una enfermedad que tiene un gran impacto en el entorno familiar de los pacientes. Esto, debido al impacto en el ámbito económico, y emocional. Por esta razón, en el manejo de pacientes con HSP es imprescindible educar a la familia en el cuidado ${ }^{11,20}$.

El personal de salud debe procurar que las familias, comprendan, conozcan y acepten la condición del paciente, que tengan claro los síntomas y las limitaciones que presentará. Esto, va a permitir que la familia se involucre en el tratamiento, siendo esta, el soporte durante todo el proceso ${ }^{20}$. Su acompañamiento, recordando la medicación, en las citas médicas, en el momento que se presenten los síntomas mantener la calma y saber actuar, y en caso de que esto no suceda comunicarse con el personal médico oportunamente. Está claro que el papel de la familia es fundamental, constituyéndose en el apoyo emocional de los pacientes para enfrentar la enfermedad de la mejor manera ${ }^{11,20}$.

\section{Conclusión}

La HSP es un evento que se presenta como respuesta fisiológica ante la lesión cerebral por diferentes causas, dada por activación simpática y catecolaminérgica. Su fisiopatología sigue en estudio aunque existen diferentes teorías pero se necesita mayor evidencia para clarificarla. El diagnóstico de HSP se hace en base a la PSH-AM donde se incorporan las manifestaciones clínicas con las herramientas de probabilidad de diagnóstico y se excluyen los diagnosticos diferenciales. Su tratamiento varía con diferentes opciones farmacológicas y no farmacológicas (como el cuidado familiar), siempre procurando mantener la estabilidad sintomatológica del paciente y evitar efectos secundarios.

Recibido: 18 de agosto de 2018 Aceptado: 24 de octubre de 2018

\section{Referencias}

1. Samuel S, Lee M, Brown RJ, Choi HA, lan J, Samuel S, et al. Incidence of paroxysmal sympathetic hyperactivity following traumatic brain injury using assessment tools. Brain Inj. 2018;00(00):[Epub ahead of print].

2. Choi HA, Jeon SB, Samuel S, Allison T, Lee K. Paroxysmal sympathetic hyperactivity after acute brain injury. Curr Neurol Neurosci Rep. 2013; 13(8).

3. Godoy DA, Panhke P, Guerrero Suarez PD, Murillo-Cabezas F. Hiperactividad simpática paroxística: Una entidad que no debería pasar desapercibida. Med Intensiva. 2017; (xx).

4. Farias-Moeller R, Carpenter JL, Dean N, Wells EM. Paroxysmal Sympathetic Hyperactivity in Critically III Children with Encephalitis and Meningoencephalitis. Neurocrit Care. 2015; 23(3): 380-5.

5. Fujiwara A, Kobata H. Paroxysmal sympathetic hyperactivity after near-hanging. Am J Emerg Med. 2015; 33(5): 735.e1-735.e2.

6. Fernández-Ortega JF, Baguley IJ, Gates TA, Garcia-Caballero M, Quesada-Garcia JG, Prieto-Palomino MA. Catecholamines and Paroxysmal Sympathetic Hyperactivity after Traumatic Brain Injury. J Neurotrauma. 2017 Jan;34(1): 109-14.

7. Letzkus L, Addison N, Turner L, Conaway M, Quatrara B. Paroxysmal Sympathetic Hyperactivity and Environmental Factors: A Pilot Study. J Neurosci Nurs. 2018; 50(2): 88-92.

8. Mathew MJ, Deepika A, Shukla D, Devi BI, Ramesh VJ. Paroxysmal sympathetic hyperactivity in severe traumatic brain injury. Acta Neurochir (Wien). 2016; 158(11): 2047-52.

9. Majdan M, Steyerberg EW, Nieboer D, Mauritz W, Rusnak M, Lingsma HF. Glasgow Coma Scale Motor Score and Pupillary Reaction To Predict Six-Month Mortality in Patients with Traumatic Brain Injury: Comparison of Field and Admission Assessment. J Neurotrauma. 2015 Jan 15; 32(2): 101-8.

10. Lump D, Moyer M. Paroxysmal sympathetic hyperactivity after severe brain injury. Curr Neurol Neurosci Rep. $2014 ; 14(11): 1-7$.

11. Meyfroidt G, Baguley IJ, Menon DK. Paroxysmal sympathetic hyperactivity: the storm after acute brain injury. Lancet Neurol. 2017; 16(9): 721-9. 
12. Moreno V, Viera R, Zárate J. Crisis Diencefálicas o Hiperactividad Simpática Paroxística: Experiencia en el Hospital Central Militar. Rev Mex Neuroci. 2017; 18(2): 118-24.

13. Meyer K. Understanding paroxysmal sympathetic hyperactivity after traumatic brain injury. Surg Neurol Int. 2014; 5(14): 490.

14. Zhang G, Li Q, Wang L, Chen Y, Zhang W, Yang H, et al. Traumatic brain injury induces macrophage subsets in the brain. Nephrol Dial Transplant. 2013; 34(1): 202.

15. Godoy DA, Pinero GR, Masotti L. Paroxysmal sympathetic hyperactivity, traumatic brain injury, and A-blockers. J Trauma Acute Care Surg. 2014; 77(2): 387-8.

16. Fernández-Ortega JF, García-Martínez MV. Furones-Lorente MJ, Quesada-García G, Prieto-Palomino MA. Paroxysmal Sympathetic Hyperactivity. J Head Trauma Rehabil. 2015; 30(5): 364-5.

17. Godo S, Irino S, Nakagawa A, Kawazoe Y, Fujita M, Kudo D, et al. Diagnosis and Management of Patients with Paroxysmal Sympathetic Hyperactivity following Acute Brain Injuries Using a Consensus-Based Diagnostic Tool: A Single Institutional Case Series. Tohoku J Exp Med. 2017; 243(1): 11-8.

18. Hughes JD, Rabinstein AA. Early diagnosis of paroxysmal sympathetic hyperactivity in the ICU. Neurocrit Care. $2014 ; 20(3): 454-9$.

19. Samuel S, Allison TA, Lee K, Choi HA. Pharmacologic management of paroxysmal sympathetic hyperactivity after brain injury. J Neurosci Nurs. 2016; 48(2): 82-9.

20. Feng $Y$, Zheng X, Fang Z. Treatment Progress of Paroxysmal Sympathetic Hyperactivity after Acquired Brain Injury. Pediatr Neurosurg. 2015; 50(6): 301-9.

21. Letzkus L, Keim-Malpass J, Kennedy C. Paroxysmal sympathetic hyperactivity: Autonomic instability and muscle over-activity following severe brain injury. Brain Inj. 2016; 30(10): 1181-5.

22. Tang Q, Wu X, Weng W, Li H, Feng J, Mao Q, et al. The preventive effect of dexmedetomidine on paroxysmal sympathetic hyperactivity in severe traumatic brain injury patients who have undergone surgery: a retrospective study. PeerJ. 2017;5: e2986.

23. Schroeppel TJ, Sharpe JP, Magnotti LJ, Weinberg JA, Clement LP, Croce MA, et al. Traumatic brain injury and $\beta$-blockers: Not all drugs are created equal. J Trauma Acute Care Surg. 2014; 76(2): 504-9.

\section{Correspondencia a:}

Dr. Luis Rafael Moscote-Salazar

mineurocirujano@aol.com 